\title{
Caracterização de usuários dos Centros de Testagem Aconselhamento no Brasil: uma revisão integrativa
}

\author{
Characterization of users of the Testing and Counseling Center in Brazil: an integrative review \\ Caracterización de los usuarios del Centro de Pruebas y Asesoramiento en Brasil: una revisión inte- \\ gradora
} Samantha Souza da Costa Pereira ${ }^{1}$, Pablo Luiz Santos Couto ${ }^{2 *}$, Marielle Maria Azevedo Silva
Rodrigues $^{3}$, Nayara Teles dos Santos ${ }^{4}$, Beatriz da Costa Pereira ${ }^{5}$, Tarcísio da Silva Flores

Como citar esse artigo. Pereira, SSC; Couto, PLS; Rodrigues, MMA; dos Santos, NT; Pereira, BC; Flores, TCS. Caracterização de usuários dos Centro de Testagem e Aconselhamento no Brasil: uma revisão integrativa. Revista Pró-UniverSUS. 2020 Jul./Dez.; 11 (2): $38-46$

\section{Resumo}

Os Centros de Testagem e Aconselhamento (CTA), são caracterizados como serviços públicos de saúde que contribuem com ações de diagnóstico e prevenção de Infecções Sexualmente Transmissíveis (IST), ademais contêm valorosa fonte de informações que viabilizam a análise e descrição do perfil social, epidemiológico e comportamental da população usuária do serviço. Objetivou-se caracterizar o perfil sociodemográfico, epidemiológico e comportamental dos usuários assistidos nos Centros Testagem e Aconselhamento. Trata-se de uma revisão integrativa de literatura, para tal foi estabelecida a questão norteadora: Qual o perfil sociodemográfico, epidemiológico e comportamental da população que utiliza os serviços oferecidos no CTA ao longo dos anos? Após a aplicação dos critérios de inclusão e exclusão, aproveitou-se 19 artigos, após pesquisa nas bases de dados Scielo, Pubmed e Biblioteca Virtual em Saúde. No Brasil, encontram-se diferenças regionais a respeito do perfil das pessoas que buscam o serviço do CTA, relacionadas ao sexo e a características socioeconômicas. Estudos apontam que o perfil dos usuários é similar em sua maioria no que diz respeito ao sexo, a faixa etária adulta, ao nível de escolaridade médio e ao não uso do preservativo em relações sexuais eventuais/fixas. Assim, conclui-se é através das ações dos CTA's que torna- se exequível acompanhar o status sorológico, o perfil dos usuários infectados e da mesma forma as variáveis da clientela usuária em cada realidade.

Palavras-chave: Centro de Testagem e Aconselhamento, Perfil epidemiológico, Infecção Sexualmente Transmissível, Saúde Coletiva, HIV.

\begin{abstract}
IThe Testing and Counseling Centers (CTA) are characterized as public health services that contribute to the diagnosis and prevention of Sexually Transmitted Infections (STI), in addition to containing a valuable source of information that enables the analysis and description of the social, epidemiological profile and behavioral of the population using the service. The objective was to characterize the sociodemographic, epidemiological and behavioral profile of users assisted at the Testing and Counseling Centers. This is an integrative literature review, for which the guiding question was established: What is the sociodemographic, epidemiological and behavioral profile of the population that uses the services offered at CTA over the years? After applying the inclusion and exclusion criteria, 19 articles were used, after searching the Scielo, Pubmed and Virtual Health Library databases. In Brazil, there are regional differences regarding the profile of people who seek the service of CTA, related to sex and socioeconomic characteristics. Studies point out that the profile of users is mostly similar with regard to sex, the adult age group, the average level of education and the non-use of condoms in casual/fixed sexual relations. Thus, it is concluded that it is through the actions of the CTA's that it becomes feasible to monitor the serological status, the profile of the infected users and in the same way the variables of the user clientele in each reality.
\end{abstract}

Keywords: Testing and Counseling Center, Epidemiological Profile, Sexually Transmitted Infection, Collective Health, HIV. 


\section{Resumen}

Los Centros de Pruebas y Asesoramiento (CTA) se caracterizan como servicios de salud pública que contribuyen al diagnóstico y la prevención de las infecciones de transmisión sexual (ITS), además de contener una valiosa fuente de información que permite el análisis y la descripción del perfil social y epidemiológico. y el comportamiento de la población que utiliza el servicio. El objetivo fue caracterizar el perfil sociodemográfico, epidemiológico y conductual de los usuarios asistidos en los Centros de Pruebas y Asesoramiento. Esta es una revisión bibliográfica integradora, para la cual se estableció la pregunta guía: ¿Cuál es el perfil sociodemográfico, epidemiológico y conductual de la población que utiliza los servicios ofrecidos en CTA a lo largo de los años? Luego de aplicar los criterios de inclusión y exclusión, se utilizaron 19 artículos, luego de buscar en las bases de datos de Scielo, Pubmed y Virtual Health Library. En Brasil, existen diferencias regionales con respecto al perfil de las personas que buscan el servicio de CTA, relacionado con el sexo y las características socioeconómicas. Los estudios señalan que el perfil de los usuarios es principalmente similar con respecto al sexo, el grupo de edad adulta, el nivel promedio de educación y la no utilización de condones en las relaciones sexuales casuales/fijas. Se concluye que es a través de las acciones de los CTA que es posible monitorear el estado serológico, el perfil de los usuarios infectados y de la misma manera las variables de la clientela del usuario en cada realidad.

Palabras clave: Centro de pruebas y asesoramiento, Perfil epidemiológico Infección Transmitida Sexualmente, Salud pública.

\section{Introdução}

Os Centros de Testagem e Aconselhamento (CTA), são caracterizados como serviços públicos de saúde que contribuem com ações de diagnóstico e prevenção de Infecções Sexualmente Transmissíveis (IST), ademais contêm valorosa fonte de informações que viabilizam a análise e descrição do perfil social, epidemiológico e comportamental da população usuária do serviço. Apresenta como propósito ampliar o acesso aos testes dessas IST's e aconselhamento a população em geral, a fim de diminuir as vulnerabilidades, o que é possível por ter caráter confidencial e resolutivo dos serviços ofertados ${ }^{1}$.

Em 1988 foi implantado o primeiro CTA no Brasil, como resposta a um problema de saúde pública com fins estratégicos para enfrentamento das IST's, implementado pela Vigilância Epidemiológica juntamente com o Ministério da Saúde (MS), o CTA dispunha dos seguintes serviços: diagnóstico precoce, prevenção e acompanhamento da doença através de testes rápidos para $\mathrm{HIV}$, garantindo aos usuários o acesso gratuito do serviço e o sigilo das informações ${ }^{2}$.

Atualmente o CTAdispõe de demais testes rápidos como: Hepatites Virais (B e C) e Sífilis, que foram aderidos ao serviço a partir da necessidade percebida nas modificações do perfil epidemiológico das IST's. O serviço inclui um caráter gratuito, sigiloso, anônimo e tem no aconselhamento uma abordagem educativa que visa à diminuição dos riscos e vulnerabilidades ${ }^{3,4}$.

De acordo com as novas condutas nacionais, o CTA tem como objetivos, favorecer e estimular o uso de medidas de prevenção; diminuir os fatores relacionados ao impacto emocional e alargar o alcance ao diagnóstico precoce da infecção pelo HIV, sífilis e hepatites $\mathrm{B}$ e C; estimular a reflexão dos usuários sobre sexualidade e estimular também o diagnóstico dos parceiros sexuais ${ }^{1,4}$.

O processo do aconselhamento pré e pós-teste realizado no CTA busca de forma individualizada ou coletiva realizar educação em saúde para os usuários do serviço, orientando-os a respeito da prevenção, tratamento e enfrentamento do possível resultado positivo dos testes. Os profissionais envolvidos no processo do aconselhamento fazem parte de uma equipe multidisciplinar, onde o foco está numa postura dialógica e de confiança entre profissionais e usuários ${ }^{3}$.

A Organização Mundial da Saúde (OMS) calcula que no mundo há mais de 1 milhão de casos de Infecções Sexualmente Transmissíveis (IST) por dia. Por ano, acredita- se que aproximadamente 357 milhões de infecções novas, entre clamídia, gonorreia, sífilis e tricomoníase. A existência de uma IST, como sífilis ou qualquer outra aumenta muito o risco do indivíduo em adquirir e/ou transmitir o HIV ${ }^{5,6}$.

De acordo com o Departamento de Infecções Sexualmente Transmissíveis (IST), AIDS e Hepatites Virais do MS presumem que nos dias atuais existam 734 mil pessoas de 15 a 49 anos vivendo com HIV/AIDS, no Brasil no ano de 2014. Dessas, em média 255 mil não sabem sua situação sorológica ${ }^{7}$.

Considerando a epidemiologia, os dados que são produzidos por este serviço (CTA) são extremamente relevantes, pois certificam a construção e implementação de estratégias de prevenção mais eficazes, além de viabilizar que as atividades assistenciais aconteçam de forma planejada e organizada ${ }^{2,8}$.

Assim, este estudo justificou-se pela necessidade em se conhecer o perfil da população que busca os serviços do CTA, a fim de inteirar-se dos principais grupos vulneráveis contribuindo para aplicação de estratégias de prevenção e tratamento para melhor atender as necessidades reais da clientela. O objetivo foi caracterizar o perfil sociodemográficos, epidemiológico e comportamental dos usuários assistidos nos Centros Testagem e Aconselhamento. 


\section{Metodologia}

Trata-se de uma revisão integrativa de literatura, método este que possibilita a súmula de conhecimento e a incorporação da aplicabilidade de resultados de estudos importantes na prática ${ }^{9}$. O desenvolvimento deste modelo prevê seis etapas, que foram utilizadas para a realização deste trabalho, a saber: 1) identificação do tema e formulação da questão norteadora, 2) busca na literatura e seleção criteriosa das pesquisas, 3) categorização dos estudos encontrados, 4) análise dos estudos incluídos, 5) interpretação dos resultados e comparações com outras pesquisas e 6) relato da revisão e síntese do conhecimento evidenciado nas pesquisas ${ }^{9}$.

Para a realização da revisão integrativa foi estabelecida a questão norteadora: Qual o perfil sociodemográfico, epidemiológico e comportamental da população que utiliza os serviços oferecidos no CTA ao longo dos anos? Foram estabelecidos como critérios de inclusão artigos com texto completo, originais, cujos dados foram coletados em campo. Do mesmo modo, serviram como fatores de exclusão, os seguintes aspectos: artigos não disponibilizados na versão completa e artigos de revisão.

Em seguida, foi realizado o levantamento de artigos científicos publicados e indexados nos bancos de dados eletrônico das últimas três décadas, Scientific Eletronic Library Online (Scielo), Pubmed e a Biblioteca Virtual em Saúde (BVS), além disso, utilizou-se a bibliografia disponibilizada pelo Ministério da Saúde em seu sítio eletrônico em busca de referências com as palavras chave: Centro de Testagem e Aconselhamento; Epidemiologia; IST's. Em seguida os artigos foram lidos na íntegra, a fim de serem selecionados aqueles que comporiam o trabalho, atendendo aos critérios estabelecidos.

Desse modo, foram encontrados 114 artigos, dos quais foram incluídos aa etapa de pré análise dos dados, 32 referências, contudo, após a leitura dos resumos, resultados e conclusões/considerações finais, foram excluídos 13 artigos, cujos temas e informações se repetiram, sendo aproveitados, portanto 19 artigos, conforme Figura 01.

Posteriormente, aplicou-se uma análise qualitativa interpretativa, iniciada com uma leitura flutuante e depois uma leitura crítica do material selecionado para classificação dos códigos e unidades de texto para a construção de inferências e interpretações. Posteriormente, foi possível a construção de uma linha do tempo, que se pautou na síntese e conteúdo semântico convergente das informações pertinentes à questão de pesquisa. A linha do tempo possibilitou a caracterização do perfil dos usuários do CTA desde a sua criação (final da década de 80) até os dias o ano de 2017.

Ressalta-se que para favorecer a integração e o agrupamento temporal dos resultados, foi construído
Figura 1. Fluxograma detalhado da seleção sistemática dos artigos incluídos no estudo. 2009 a 2019. Guanambi, Brasil, março de 2020.

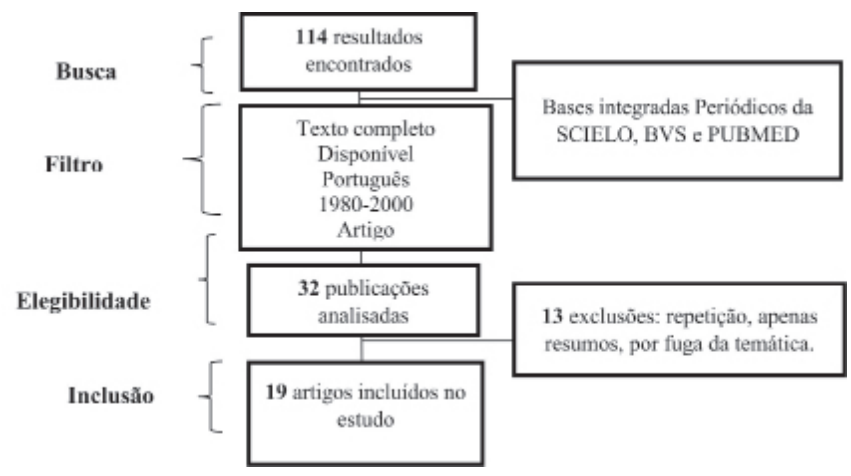

Fonte: Dados do Estudo

um quadro sinóptico integrativo, cujo intuito foi sintetizar as informações mais relevantes dos artigos que atenderam aos critérios de inclusão, bem como, facilitar a visualização e sintetizar os resultados dos artigos.

Por se tratar de uma pesquisa que utiliza como fonte de dados uma base secundária e de acesso público, não se faz necessário à aprovação de um Comitê de Ética em Pesquisa para a realização do estudo.

\section{Resultado}

Abaixo, quadrosinóptico, com a síntese dos artigos selecionados que atenderam aos critérios de inclusão. Após análise dos resultados e a sua interpretação, a linha do tempo possibilitou três categorias: Usuários do COAS nos anos 80; Usuários do CTA nos anos 90; Perfil dos usuários nos anos 2000.

\section{Discussão}

As IST's são classificadas como uma das dificuldades de saúde pública mais habitual em todo o mundo. Independentemente de sexo, transforma o organismo mais suscetível a outras doenças, inclusive o HIV/AIDS. No Brasil, temos os seguintes dados anualmente: Sífilis: 937.000; Gonorreia: 1.541.800; Clamídia: 1.967.200; Herpes genital: 640.900, HPV: 685.4007.

A epidemia das IST's é vista como um evento complexo, instável e ativo constituindo um relevante problema de saúde pública em todo o mundo. Acreditase que várias questões influem na sua ocorrência, dentre elas as que são mais evidentes são os referentes ao comportamento humano tanto de forma individual como coletiva ${ }^{2}$.

A ampliação da epidemia de AIDS carrega consigo a oportunidade da prática de realização de exames sorológicos, o que possibilitou o diagnóstico da infecção pelo HIV a um número progressivo de pessoas. 
Quadro 1. Quadro sinóptico com a inclusão dos artigos incluídos no estudo, por ordem de publicação. Guanambi, Bahia, Brasil. 2018

\begin{tabular}{|c|c|c|}
\hline AUTORES & ARTIGO & RESULTADOS \\
\hline $\begin{array}{l}\text { Bassicheto et } \\
\text { al. (2004) }\end{array}$ & $\begin{array}{l}\text { Perfis epidemiológicos dos usu- } \\
\text { ários de um Centro de Testagem } \\
\text { e Aconselhamento para DST/ } \\
\text { HIV da Rede Municipal de São } \\
\text { Paulo, com sorologia positiva } \\
\text { para o HIV. }\end{array}$ & $\begin{array}{l}\text { Predominância do sexo masculino, Brancos, Nível de escolaridade médio incompleto, } \\
\text { Solteiros } \\
\text { Comportamento de risco homossexualidade, Parceiros múltiplos, Não uso do preserva- } \\
\text { tivo }\end{array}$ \\
\hline $\begin{array}{l}\text { Wolffenbüttel } \\
\text { e Carneiro. } \\
\quad(2007)\end{array}$ & $\begin{array}{l}\text { Uma breve história dos Centros } \\
\text { de Testagem e Aconselhamento } \\
\text { (CTA) enquanto organização } \\
\text { tecnológica de prevenção de } \\
\text { DST/Aids no Brasil e no estado } \\
\text { de São Paulo }\end{array}$ & $\begin{array}{l}\text { A comparação entre } 2001 \text { e } 2005 \text {, mostra o aumento da proporção de CTA inseridos em } \\
\text { unidades de saúde, e o aumento na oferta de exames sorológicos, particularmente para } \\
\text { hepatites virais B e C. A heterogeneidade no perfil dos CTA do Estado de São Paulo, } \\
\text { provavelmente, ocorre também em outros estados. } \\
\text { As diretrizes inclusão do auxílio aos usuários no processo de adesão aos tratamentos } \\
\text { anti-retrovirais: o estímulo do diagnóstico das parcerias sexuais; o auxílio dos serviços de } \\
\text { pré-natal para a testagem sorológica de gestantes; e o oferecimento de informações sobre } \\
\text { prevenção das DST/HIV/Aids e do uso indevido de drogas para grupos específicos. }\end{array}$ \\
\hline $\begin{array}{l}\text { Matos et al. } \\
(2011)\end{array}$ & $\begin{array}{l}\text { Perfis Sociocomportamentais } \\
\text { dos Usuários do Centro de Tes- } \\
\text { tagem e Aconselhamento- CTA } \\
\text { em Dst/Aids do Hospital Uni- } \\
\text { versitário Getúlio Vargas da Ci- } \\
\quad \text { dade de Manaus-AM. }\end{array}$ & $\begin{array}{l}\text { Predomínio do sexo feminino, Faixa etária adulta, Casada, Nível de escolaridade médio, } \\
\text { Motivo de procura a prevenção. } \\
\text { Comportamento de risco bissexualidade, Não uso do preservativo, Procurou o serviço } \\
\text { por prevenção }\end{array}$ \\
\hline $\begin{array}{l}\text { Souza et al. } \\
\qquad(2011)\end{array}$ & $\begin{array}{l}\text { Perfil da população idosa que } \\
\text { procura o centro de referência } \\
\text { em DST/Aids de Passos/MG. }\end{array}$ & $\begin{array}{l}\text { Sexo masculino, Faixa etária de } 60-65 \text { anos, Nível de escolaridade médio, Casado } \\
\text { Comportamento sexual Heterossexual, Não uso do preservativo, Procurou o serviço por } \\
\text { risco de exposição a IST's }\end{array}$ \\
\hline $\begin{array}{l}\text { Soares et al. } \\
(2013)\end{array}$ & $\begin{array}{l}\text { Não retorno de usuários a um } \\
\text { Centro de Testagem e Aconse- } \\
\text { lhamento do Estado do Rio de } \\
\text { Janeiro: fatores estruturais e } \\
\text { subjetivos. }\end{array}$ & $\begin{array}{l}\text { Entre } 2008-2009,62 \% \text { dos usuários, excluindo-se as gestantes, não resgataram resultados } \\
\text { de seus testes nesse CTA. As circunstâncias que determinam o não retorno referem-se ao } \\
\text { acolhimento precário ao usuário e não garantia de direitos como anonimato e confiden- } \\
\text { cialidade, às dificuldades operacionais (como atraso na entrega de resultados) e também } \\
\text { medo, ansiedade, representações negativas da testagem, discriminação. A maioria era do } \\
\text { sexo feminino }(62,5 \%) \text {, parda }(41,8 \%) \text {, com idade entre } 20 \text { e } 34 \text { anos }(40 \%) \text {, casados/ } \\
\text { amigados }(49,9 \%) \text {, de escolaridade média-alta2 }(50,5 \%) \text { e heterossexuais (aproximada- } \\
\text { mente } 90 \%) \text {. }\end{array}$ \\
\hline $\begin{array}{l}\text { Vilela et al. } \\
\qquad(2014)\end{array}$ & $\begin{array}{l}\text { Perfil epidemiológico dos usu- } \\
\text { ários do Centro de Testagem e } \\
\text { Aconselhamento de Alfenas, } \\
\text { Minas Gerais. }\end{array}$ & $\begin{array}{l}\text { Predominância do sexo feminino, População adulta jovem, Solteiras, Nível de escolari- } \\
\text { dade médio, Brancas } \\
\text { Relacionamento sexual homossexual, Não uso de preservativos, Procurou o serviço pelo } \\
\text { risco de exposição a IST's }\end{array}$ \\
\hline $\begin{array}{l}\text { Brito et al. } \\
\quad(2014)\end{array}$ & $\begin{array}{l}\text { Perfil epidemiológico de por- } \\
\text { tadores do vírus da imunodefi- } \\
\text { ciência humana e síndrome da } \\
\text { imunodeficiência adquirida no } \\
\text { estado de Sergipe, 2007-2012. }\end{array}$ & $\begin{array}{l}\text { Predominância do sexo masculino, Faixa etária de } 21-50 \text { anos, Nível de escolaridade } \\
\text { básico/fundamental } \\
\text { Parceiros fixos e não fixos, Não uso do preservativo, Heterossexualidade como padrão } \\
\text { da infecção. }\end{array}$ \\
\hline $\begin{array}{l}\text { Nascimento et } \\
\text { al. (2014) }\end{array}$ & $\begin{array}{l}\text { Aspectos sociodemográficos } \\
\text { e comportamentais dos usuá- } \\
\text { rios de um Centro de Testagem } \\
\text { e Aconselhamento para DST/ } \\
\text { AIDS da Rede Municipal de Be- } \\
\text { lém, Pará, com sorologia positi- } \\
\text { va para o HIV. }\end{array}$ & $\begin{array}{l}\text { Predominância do sexo masculino, Faixa etária adulta, Solteiros, Pardos } \\
\text { Nível de escolaridade médio, Relações sexuais heterossexuais, Não uso do preservativo }\end{array}$ \\
\hline $\begin{array}{l}\text { Barbosa et al., } \\
\quad(2016)\end{array}$ & $\begin{array}{c}\text { Behavioral profile of pregnant } \\
\text { women attended in a testing and } \\
\text { counseling center in a frontier } \\
\text { municipality. }\end{array}$ & $\begin{array}{l}\text { Gestantes na faixa etária de } 20 \text { a } 29 \text { anos, casadas, brancas, tinham menos de } 11 \text { anos de } \\
\text { estudo. } \\
\text { Procuraram o CTA no segundo trimestre de gestação e apenas uma parcela muito peque- } \\
\text { na pertenciam a classes de comportamento de risco para contrair DST (profissional do } \\
\text { sexo e usuários de drogas). } \\
\text { Sobre o uso de preservativos com parceiros eventuais, a maioria preferiu não responder, } \\
\text { inclusive sobre possíveis motivos. }\end{array}$ \\
\hline $\begin{array}{l}\text { Ferreira et al. } \\
\qquad(2016)\end{array}$ & $\begin{array}{l}\text { Perfil epidemiológico dos usuá- } \\
\text { rios de um centro de testagem e } \\
\text { aconselhamento da Bahia. }\end{array}$ & $\begin{array}{l}\text { Predomínio de mulheres }(52,87 \%) \text {, faixa etária entre } 20 \text { a } 30 \text { anos }(40,32 \%) \text {, casados/ } \\
\text { união estável }(48,69 \%) \text { e escolaridade de } 8 \text { a } 11 \text { anos }(38,14 \%) \text {. } \\
\text { A prevalência de infecções sexualmente transmissíveis foi } 12,25 \% \text {, mais frequente entre } \\
\text { os homens, e o agravo mais prevalente foi sífilis }(4,52 \%) \text {, seguido de infecção por HIV } \\
(3,33 \%) \text {, hepatites B }(2,62 \%) \text { e hepatite C }(1,78 \%) \text {. } \\
\text { O uso consistente do preservativo com parceiro fixo }(17,19 \%) \text { e parceiro não fixo } \\
(34,37 \%) \text { foi baixo, com maior exposição do sexo feminino. }\end{array}$ \\
\hline
\end{tabular}


Quadro 1 (cont.). Quadro sinóptico com a inclusão dos artigos incluídos no estudo, por ordem de publicação. Guanambi, Bahia, Brasil. 2018

\begin{tabular}{|c|c|c|}
\hline AUTORES & ARTIGO & RESULTADOS \\
\hline $\begin{array}{l}\text { Bones-Rocha } \\
\text { et al. (2017) }\end{array}$ & $\begin{array}{c}\text { Impacto de la implementación } \\
\text { de la prueba rápida del VIH/ITS } \\
\text { en un centro de atención espe- } \\
\text { cializada en Brasil }\end{array}$ & $\begin{array}{l}\text { Maioria homens e brancos, Solteiros, } 07 \text { anos de estudo. (nível de escolaridade) } \\
35 \% \text { apenas usa preservativo nas relações. }\end{array}$ \\
\hline $\begin{array}{l}\text { Segundo et al. } \\
\qquad(2017) \text {. }\end{array}$ & $\begin{array}{c}\text { Fatores associados às infecções } \\
\text { sexualmente transmissíveis em } \\
\text { usuários de centro de testagem e } \\
\text { aconselhamento da Bahia }\end{array}$ & $\begin{array}{l}\text { Prevalência de Infecções Sexualmente Transmissíveis de } 11,1 \% \text {. } \\
\text { O HIV foi o agravo mais prevalente }(4,2 \%) \text {, seguido da sífilis }(4 \%) \text { e hepatites B }(2,1 \%) \\
\text { e C }(2,1 \%) \text {. } \\
\text { O uso consistente de preservativo foi baixo, com parceiros fixos }(9,5 \%) \text { e não fixos } \\
(16,2 \%) \text {. } \\
\text { Maioria do sexo masculino, com idade de } 20 \text { a } 50 \text { anos, pardos, com } 1 \text { a } 7 \text { anos de es- } \\
\text { tudo }\end{array}$ \\
\hline $\begin{array}{l}\text { Nogueira et } \\
\text { al. (2017) }\end{array}$ & $\begin{array}{l}\text { Caracterização dos usuários } \\
\text { atendidos em um centro de tes- } \\
\text { tagem e aconselhamento em in- } \\
\text { fecções relacionadas ao sexo }\end{array}$ & $\begin{array}{l}15.836 \text { usuários realizaram a testagem rápida. } \\
\text { Constatou-se que os motivos que os levaram a buscar o CTA foram exposição a situações } \\
\text { de risco } 68,7 \% \text {, sendo que, da totalidade, no público masculino os índices de resultados } \\
\text { positivos foram o de HIV, com } 3,6 \% \text {, e sífilis, com } 3,9 \% \text {. } \\
\text { Além disso, } 32,5 \% \text { da população estudada relatou usar preservativos com parceiros fi- } \\
\text { xos. }\end{array}$ \\
\hline $\begin{array}{l}\text { Barros et al. } \\
(2017)\end{array}$ & $\begin{array}{l}\text { Individual- and contextual-level } \\
\text { factors associated with client- } \\
\text { initiated HIV testing }\end{array}$ & $\begin{array}{l}5.040 \text { brasileiros, composta por } 2.298 \text { homens e } 2.742 \text { mulheres, com idades entre } 16 \text { e } \\
65 \text { anos, residentes em áreas urbanas. } \\
\text { Apenas dados de indivíduos sexualmente ativos foram incluídos. } \\
\text { Sexo feminino, } 70,3 \% \text {, idade } \geq 45 \text { anos, } 87,5 \% \text {, branca ou parda, } 45,1 \% \text { possuíam ensino } \\
\text { fundamental. }(61,6 \%) \text { era casada ou morava com companheiro e }(64,2 \%) \text { relatou ser } \\
\text { católica. } \\
(60,2 \%) \text { relatou ter relações sexuais não forçadas pela primeira vez aos } 15 \text { anos, ser hete- } \\
\text { rossexual ( } 98 \%) \text {, não ter DST ( } 82,1 \% \text { ) ou ter vivenciado uma situação de violência (93,6 } \\
\%) 32,1 \% \text { não usaram camisinha no primeiro momento e } 60,2 \% \text { na última relação sexual, } \\
62,3 \% \text { tiveram mais de três parceiros sexuais e } 45,1 \%\end{array}$ \\
\hline $\begin{array}{l}\text { Araújo et al. } \\
\quad(2018)\end{array}$ & $\begin{array}{c}\text { Self-care of elderly people after } \\
\text { the diagnosis of acquired immu- } \\
\text { nodeficiency syndrome }\end{array}$ & $\begin{array}{l}\text { Os dados mostram o (des) conhecimento por parte dos idosos acerca da transmissão do } \\
\text { HIV/Aids, a vivência da condição de ser idoso e ter HIV/Aids, o cuidado de si e como é } \\
\text { a vida após o diagnóstico de HIV/Aids em seu cotidiano. } \\
\text { A maioria era do sexo feminino, com faixa etária de } 60 \text { a } 70 \text { anos. } \\
\text { A maioria iria ao CTA, mas desconhecia o uso do preservativo. }\end{array}$ \\
\hline $\begin{array}{l}\text { C l a u d i a n o } \\
\text { e } \quad \text { Moraes } \\
(2019)\end{array}$ & $\begin{array}{l}\text { Sociodemographic and behavio- } \\
\text { ral profile of users testing and } \\
\text { counseling center in DST/Aids. }\end{array}$ & $\begin{array}{l}74,7 \% \text { eram do sexo masculino, } 77,6 \% \text { eram solteiros e a idade predominante era de } 18 \\
\text { a } 24 \text { anos para ambos os sexos. } \\
\text { A relação sexual foi a principal forma de exposição ao risco: } 9,8 \% \text { apresentou algum } \\
\text { tipo de horário de verão nos últimos } 12 \text { meses e, desses, } 30,9 \% \text { não tiveram nenhum } \\
\text { tratamento. } \\
\text { Os trabalhadores do sexo representam } 3,7 \% \text { dos usuários e homens que fizeram sexo com } \\
\text { homens (HSH) representam } 43 \% \text {. } \\
\text { Os preservativos foram usados nas relações sexuais com parceiros casuais em todas as } \\
\text { ocasiões em } 47,6 \% \text {, enquanto } 11,6 \% \text { não usaram preservativos em nenhuma ocasião. }\end{array}$ \\
\hline $\begin{array}{l}\text { Ferreira et al. } \\
\qquad(2019)\end{array}$ & $\begin{array}{l}\text { Vulnerabilidade a infecções se- } \\
\text { xualmente transmissíveis em } \\
\text { idosos usuários de um centro de } \\
\text { testagem e aconselhamento }\end{array}$ & $\begin{array}{l}\text { Sexo masculino (60,94\%), faixa etária de } 60 \text { a } 70 \text { anos }(75,97 \%) \text {, cor parda }(26,61 \%) \text {, } \\
\text { casados }(61,80 \%) \text {, aposentados }(57,08 \%) \text { e com escolaridade de } 4 \text { a } 7 \text { anos de estudo } \\
(35,19 \%) \text {. } \\
\text { A maioria relatou a relação sexual como tipo de exposição }(76,39 \%) \text {, preferência heteros- } \\
\text { sexual }(92,27 \%) \text { e parceria fixa }(72,96 \%) \text {. } \\
\text { A frequência de uso do preservativo foi baixa com o parceiro não fixo }(32,73 \%) \text { e com o } \\
\text { parceiro fixo }(5,58 \%) \text {. } \\
\text { A prevalência de IST foi } 25,32 \% \text {, com maior percentual entre os homens. A IST mais } \\
\text { prevalente foi a hepatite C }(10,73 \%) \text {, seguida da hepatite B }(8,58 \%) \text {, sífilis }(7,73 \%) \text { e } \\
\text { HIV }(3,43) \text {. }\end{array}$ \\
\hline Melo (2020) & $\begin{array}{l}\text { Ações itinerantes do Centro de } \\
\text { Testagem e Aconselhamento } \\
\text { (CTA) em ambiente universitá- } \\
\text { rio }\end{array}$ & $\begin{array}{l}\text { CTA itinerante: } 2011 \text { e 2012, a maioria foi predominantemente do sexo feminino, em } \\
2013 \text { houve uma modificação no perfil, com a quantidade de homens superando o de } \\
\text { mulheres. A maioria ao longo desse período sempre teve parceiros fixos. } \\
\text { Quanto ao uso de preservativo } 2011 \text { a maioria não fazia uso do preservativo, havendo } \\
\text { modificação em } 2012 \text { e 2013, com a maioria fazendo uso do preservativo. }\end{array}$ \\
\hline
\end{tabular}


Quadro 1 (cont.). Quadro sinóptico com a inclusão dos artigos incluídos no estudo, por ordem de publicação. Guanambi, Bahia, Brasil. 2018

\begin{tabular}{||c|c|l|}
\hline AUTORES & ARTIGO & \multicolumn{1}{c|}{ RESULTADOS } \\
\hline Lima et al. & $\begin{array}{l}\text { Percepção dos profissionais de } \\
\text { saúde e dos usuários sobre o } \\
\text { aconselhamento no teste rápido } \\
\text { para HIV }\end{array}$ & $\begin{array}{l}\text { Maioria (68,1\%) de mulheres; com relação à idade, houve variação de } 18 \text { a } 50 \text { anos (mé- } \\
\text { dia de 30,0); 78,6\% se autodeclaram pardos e 21,4\% brancos. } \\
\text { Em relação à escolaridade, 68,2\% haviam concluído o ensino médio, 22,7\% se encontra- } \\
\text { vam cursando o ensino superior e 9,1\% possuíam ensino fundamental }\end{array}$ \\
\hline
\end{tabular}

Fonte: Dados do Estudo.

Nesse contexto encontra-se a idealização e criação do CTA, que iremos discutir adiante ${ }^{10,11}$.

\section{Usuários do COAS nos anos 80}

Desde 1980, ano em que foi apontada a primeira ocorrência de AIDS no mundo, presenciou-se um frenético avanço do crescimento dessa epidemia, compenetrada em comunidades susceptíveis como profissionais do sexo, com a prática de sexo anal, gays, travestis e transexuais ${ }^{12}$. Acreditava-se que a infecção do HIV estava ligada ao estilo ou hábito de vida e a partir dessa concepção surgiu à noção de que existia um grupo considerado de risco ${ }^{13,14}$.

É no final da década de 80 que se tem o impulso inicial para a organização dos Centros de Orientação e Apoio Sorológico (COAS), assim nomeado na época, julgada como modelo original de serviços de saúde, oferecendo sorologia anti-HIV de forma gratuita, sigilosa e anônima, além de promover educação em saúde e orientação para os usuários sob ameaça de infecção pelo HIV ${ }^{1,10,13}$.

No Brasil, os exames sorológicos para identificação de anticorpos do HIV foram propostos inicialmente, em triagem de sangue para transfusões ou para fracionamento e produção de sangue e seus derivados, assim como para triagem dos doadores de órgãos e sêmen, a fim de determinar a predominância da infecção na população e para comprovar o diagnóstico das diversas formas clínicas de $\operatorname{AIDS}^{1,10}$.

Num cenário onde o conhecimento sobre a AIDS era escasso, o preconceito contra as pessoas infectadas era intenso, os serviços oferecidos pelo COAS possibilitaram novas descobertas na prática dos serviços de saúde, como a possibilidade de realização da testagem de forma anônima, gratuita e a implementação do aconselhamento como pilar central das suas atribuições ${ }^{3}$.

Utilizando- se como embasamento o direcionamento dado pelo serviço de saúde aos chamados grupos de risco, da época, pode-se inferir que o perfil da população atendida no COAS, abrangia justamente esse determinado grupo de risco, a saber: gays, transexuais, travestis (homens) e profissionais do sexo.

\section{Usuários do CTA nos anos 90}

Decorridos os anos, importantes mudanças foram consideradas, dentre elas a contribuição dos COAS na redução dos riscos em contrair o vírus do HIV, tendo também como direcionamento o atendimento a demanda espontânea para realização de orientações e educação em saúde a respeito da prevenção e diagnóstico precoce de outras IST's além do HIV ${ }^{4,8,15}$. Com o aparecimento de novos eventos de AIDS entre heterossexuais e crianças/recém nascidos, foi-se descartando a ideia de que a infecção estava vinculada somente aos indivíduos que se encaixavam no grupo de $\operatorname{risco}^{13,16}$.

Houve então a necessidade de esclarecer à população de que a infecção pelo HIV/AIDS não excluía o risco de qualquer indivíduo que adotasse um comportamento que o expusesse ao vírus de contraílo. Dessa forma o termo grupo de risco foi substituído por um mais abrangente que se diz "comportamento de risco" em relação a uma relevante quantidade de contaminação pela aplicação de drogas injetáveis, alcançando uma grande parcela de heterossexuais ${ }^{17,18}$.

Em 1997, a Coordenação Nacional de DST/ AIDS renomeou os Centros de Orientação e Apoio Sorológico (COAS) com o nome de Centro de Testagem e Aconselhamento (CTA), mudança esta que respondeu à reflexão sobre os princípios que deveriam direcionar a implementação e estabilização desses serviços ${ }^{15,18}$.

Em 1999, foram publicadas as Diretrizes dos CTA's, que estabelecem como metas para a atualização do mesmo: ampliar o alcance ao diagnóstico da infecção pelo HIV; favorecer para a diminuição de riscos de transmissão do HIV; encorajar a adoção de hábitos sexuais seguros; direcionar os usuários HIV- positivo para os serviços de referência, auxiliando-os no processo de adesão aos tratamentos antirretrovirais; receber a procura por testes sorológicos nos bancos de sangue; incentivar o diagnóstico das parcerias sexuais; amparar os serviços de pré-natal para a testagem sorológica de gestantes; expandir as informações sobre prevenção das DST/HIV/ AIDS e do uso impróprio de drogas para grupos específicos ${ }^{15,19}$.

Nesta década, à medida que a testagem foi amplificada, tanto em direção aos grupos de alta prevalência quanto às populações de baixa prevalência 
para a infecção pelo HIV, percebe-se que aos poucos o perfil dos usuários se modifica, deixando que o foco do serviço seja apenas para as classes consideradas de alta prevalência, como homossexuais. Reconhecendo novos grupos populacionais que estavam expostos ao risco de contaminação, como as gestantes ampliando os serviços e trabalhando com o conceito de referência e contra referência ${ }^{1,2} 0$.

A incorporação das gestantes na realização dos testes rápidos é um avanço e um marco importante no que diz respeito à transmissão de IST's por via materna, pois com o diagnóstico precoce através do pré- natal é possível tratar a gestante em tempo hábil a fim de diminuir ou anular os riscos de contaminação para o feto e/ou recém- nascido.

\section{Perfil dos usuários nos anos 2000}

No ano de 2001 foi elaborado o Sistema de Informação de CTA (SI-CTA) proposto a equipar técnicos e gestores dos CTA e das coordenações nas três esferas de governo, de DST/AIDS no atendimento ao cliente e no gerenciamento tanto de recursos humanos como materiais, além de integrar o sistema de vigilância da infecção pelo HIV e de proporcionar a realização de investigações específicas. Concomitantemente, os CTA' s são solicitados a oferecer testagem sorológica para as hepatites $\mathrm{B}$ e C, com aconselhamento pré e pósteste $^{15,21}$.

No Brasil, encontram-se diferenças regionais a respeito do perfil das pessoas que buscam o serviço do CTA, relacionadas ao sexo e a características socioeconômicas. A procura de forma espontânea ainda é muito baixa na maioria dos CTA; grande parte dos usuários que procuram o serviço o faz por ter vivido alguma situação relacionada ao risco de infecção ${ }^{1-2,22}$.

Os aspectos relacionados ao processo do serviço são motivadores importantes para a não adesão ao serviço de forma completa ${ }^{7}$. O modelo de uso do serviço de saúde está relacionado de forma predominante pelo paciente, nos casos em que a busca se dá de forma espontânea, por exemplo, a utilização será determinada pelas particularidades de quem busca o serviço e também das questões relacionadas à estrutura do sistema de saúde ${ }^{23}$.

As questões estruturais têm forte influência na adesão/ procura do cliente ao serviço, uma vez que a aparência das instituições e organizações de saúde é levada em consideração pelo usuário. Outro fator importante relacionado à estrutura do CTA é a má localização, escassez na divulgação e a falta de placas de direcionamento para o serviço ${ }^{1,4}$. A disponibilização dos materiais necessários para a prevenção e diminuição de danos no CTA deve contar com o reconhecimento do perfil da população usuária do serviço, fazendo com que sejam disponibilizados insumos que supram a necessidade real da clientela, garantindo assim o acesso facilitado dos usuários aos materiais ${ }^{22-24}$.

Dessa forma, alguns estudos realizados acerca do tema apontam as características dos usuários desse serviço, de forma que torna possível a identificação das similaridades e diferenças regionais do perfil deste usuário $^{2-4,14,16}$. Em uma pesquisa desenvolvida em Manaus, a fim de conhecer o perfil dos usuários do CTA tendo como resultado: predomínio do sexo feminino, sendo a faixa etária entre 20 a 39 anos, casada, com nível de escolaridade variando entre oito e 11 anos de estudo concluídos. Tendo como principal motivo de procura a prevenção. Em relação ao comportamento de risco, grande parte referiu relação bissexual e o não uso do preservativo tanto nas relações fixas como eventuais ${ }^{24}$.

Contudo, em outro estudo realizado, houve a caracterização do perfil dos usuários idosos que frequenta o CTA e revelou que grande parte deste são do sexo masculino, na faixa etária de 60-65 anos, com ensino fundamentou e médio completos, casados, heterossexuais e que procuram o serviço por algum tipo de exposição a IST' $\mathrm{s}$, sendo que relataram ter atividade sexual desprotegida ${ }^{25}$. Também identificouse um aumento considerável no número da procura ao serviço do CTA pelos idosos no ano de 2007, o que foi justificado pelo empenho do MS nas campanhas de prevenção voltadas a esse grupo ${ }^{25}$. Tal constatação mostra a importância em se reconhecer o perfil dos usuários do serviço do CTA, a fim de direcionar políticas públicas ao público alvo identificado ${ }^{23,26}$.

Segundo a pesquisa realizada em Alfenas, Minas Gerais, o perfil dos usuários do CTA é caracterizado pela predominância do sexo feminino, fator que foi relacionado pelo autor ao fato de que este sexo preocupase mais com a saúde, pela faixa etária de 20 a 29 anos, uma população adulta jovem. No que diz respeito ao estado civil, foi identificado um predomínio de usuários solteiros, com 12 anos ou mais de escolaridade e que se consideram brancos ${ }^{27}$.

$\mathrm{O}$ motivo mais frequente quanto à procura do serviço é o risco da exposição a alguma IST, justificado pelo não uso do preservativo pela maioria dos usuários, o tipo de parceria sexual foi de relacionamentos homossexuais e o tipo de IST mais prevalente foram as hepatites $\mathrm{B} \mathrm{e} \mathrm{C}^{28}$. Pelo fato de um dos objetivos iniciais do CTA ser o combate e prevenção ao HIV/AIDS, existem mais estudos que relatam as características epidemiológicas e comportamentais dos usuários com a presença do vírus do HIV no organismo que o perfil dos usuários de forma geral ${ }^{26-28}$.

$\mathrm{Na}$ pesquisa realizada nos CTA's da rede municipal de São Paulo, mencionou-se as características do perfil dos usuários portadores do HIV em um CTA, e verificou-se o seguinte: predominância do sexo masculino, faixa etária de 32 anos, brancos, com nível 
de escolaridade médio incompleto, solteiros e relatam risco de exposição sexual homossexual e parceiros múltiplos. Confrontando os resultados detectados como reagentes para HIV, em sua maioria, também o foram para sífilis ${ }^{22}$.

Favorecendo o que foi encontrado em outras pesquisas são concernentes em relação ao que foi encontrado em suas pesquisas, no que diz respeito ao sexo, a faixa etária adulta, ao nível de escolaridade e ao não uso do preservativo em relações sexuais eventuais/ fixas ${ }^{2,18,22}$. Contradizendo no aspecto da exposição homossexual, a heterossexualização é vista como padrão atual da infecção e o seu contágio se da através dos comportamentos sexuais ${ }^{26,28}$.

\section{Considerações Finais}

Conclui-se que a linha do tempo revelada na literatura, permite a inferência de que, com o passar dos anos houve um aumento gradativo no número de pessoas que buscam pelo serviço do CTA, ao passo que as orientações a respeito da prevenção e diagnóstico precoce das IST's foram se fortalecendo ao longo dos anos e disseminadas nos meios de comunicação e mídia (ainda que de modo incompleto).

Contudo, tornou-se possível evidenciar a importância dos serviços oferecidos pelo CTA na prevenção e diagnóstico precoce das IST' $\mathrm{s}$ assim como a relevância em se conhecer o perfil dos usuários deste serviço em cada região. Pois é através das ações dos CTA' s que torna- se exequível acompanhar o status sorológico, o perfil dos usuários infectados e da mesma forma as variáveis da clientela usuária em cada realidade.

Destarte, assim como existem características similares entre os usuários encontram-se diferenças regionais que necessitam ser ponderadas para que estratégias e condutas de prevençãoe adesão da população ao serviço sejam tomadas de forma direcionada a cada necessidade. Além disso, as reflexões aqui apresentadas poderão favorecer a adoção de estratégias de promoção à saúde por profissionais de saúde, no que tange a saúde sexual e a prevenção das IST's/aids.

Consequentemente, as condutas e orientações realizadas no aconselhamento pré e pós teste devem guiar-se de maneira tal que as informações disseminadas cheguem ao usuário de forma clara e objetiva respeitando as limitações intelectuais, sociais e comportamentais do mesmo. A fim de diminuir os riscos, prevenir, controlar e interromper a cadeia de transmissão das IST's/AIDS.

\section{Referências}

11. Brasil. Ministério da Saúde. Secretaria de Vigilância em Saúde. Departamento de DST, AIDS e Hepatites virais. Diretrizes para organização e funcionamento dos CTA no Brasil: Manual. Brasília, DF: Ministério da
Saúde, 2010. Available from: http://www.aids.gov.br/pt-br/pub/2010/ diretrizes-para-organizacao-e-funcionamento-dos-cta

2. Nascimento RG, Sousa RCM, Pinto DS. Aspectos sociodemográficos e comportamentais dos usuários de um Centro de Testagem e Aconselhamento para DST/AIDS da Rede Municipal de Belém, Pará, com sorologia positiva para o HIV. Rev Epidemiol Control Infect. 2014;4(2):132-138. doi: http:// dx.doi.org/10.17058/reci.v4i2.4210

3. Monteiro SS, Brandão E, Vargas E, Mora C, Soares P, Daltro E. Discursos sobre sexualidade em um Centro de Testagem e Aconselhamento (CTA). Ciênc saúde Coletiva. 2014;19(1):137-146. doi: https://doi. org/10.1590/1413-81232014191.1906

4. Lima PBSXC, Araújo MAL, Melo AK, Leite JMA. Percepção dos profissionais de saúde e dos usuários sobre o aconselhamento no teste rápido para HIV. Esc Anna Nery. 2020;24(2): e20190171. doi: http://dx.doi org/10.1590/2177-9465-ean-2019-0171.

5. Brasil. Ministério da Saúde. Secretaria de Vigilância em Saúde, PN de DST e AIDS. Boletim Epidemiológico HIV/AIDS. Ano V(1): $1^{\text {a }}$ à $26^{\text {a }}$ semanas epidemiológicas, jan./jun. Brasília: Ministério da Saúde, 2016. Available from: http://www.aids.gov.br/pt-br/pub/2016/boletimepidemiologico-de-aids-2016

6. Bones-Rocha K, Carvalho FT, Almeida-Weber JL, Silvana-Both N, Pizzinato A. Impacto de la implementación de la prueba rápida del VIH/ITS en un centro de atención especializada en Brasil. Revista Gerencia y Políticas de Salud. 2017;16(32):108-119. https://dx.doi.org/10.11144/javeriana. rgps16-32.iipr

7. Brasil. Secretaria de Vigilância em Saúde, PN de DST e AIDS. Boletim Epidemiológico HIV/AIDS. Ano III(1): $1^{\mathrm{a}}$ à $26^{\mathrm{a}}$ semanas epidemiológicas, jan./jun. Brasília: Ministério da Saúde, 2014. Available from: http://www. aids.gov.br/pt-br/node/73

8. Claudiano FS, Moraes JC. Sociodemographic and behavioral profile of users testing and counseling center in DST/Aids. Biosci. J. 2019;35(1):326332. http://dx.doi.org/10.14393/BJ-v35n1a2019-33344

9. Neto NTA, Constantino P, Assis SG. Análise bibliográfica da produção em saúde sobre adolescentes cumprindo medidas socioeducativas de privação de liberdade. Physis. 2017;27(3):511-40. Disponível em: http:/ www.scielo.br/pdf/physis/v27n3/1809-4481-physis-27-03-00511.pdf

10. Brasil. Ministério da Saúde. Coordenação Nacional de DST e Aids. Diretrizes dos Centros de Testagem e Aconselhamento - CTA: Manual. Brasília, DF: Ministério da Saúde, 1999. Available from: http://bvsms.saude. gov.br/bvs/publicacoes/cd03_17.pdf

11. Ferreira CO, Davoglio RS, Silva AA, Viana ASA, Gomes AVTM. Perfil epidemiológico dos usuários de um centro de testagem e aconselhamento da Bahia. Rev baiana Saud Pública. 2016;40(2). doi: https:// doi.org/10.22278/23182660.2016.v40.n2.a1980

12. Tokano DV, Dessunti EM. Centro de testagem e aconselhamento: características dos usuários e prevalência de infecção pelo HIV. Ciência, Cuidado e Saúde. 2016;14(4):1537-1545. Available from: https://doi. org/10.4025/cienccuidsaude.v14i4.22441

13. Silva TQC, Szapiro AM. Mulheres heterossexuais em relacionamento estável: limites do aconselhamento em DST/HIV/AIDS. Rev Subjetividades. 2015;15(3):350-361. Available from: http://pepsic.bvsalud.org/pdf/rs/ v15n3/04.pdf

14. Melo W. (2019). Ações itinerantes do Centro de Testagem e Aconselhamento (CTA) em ambiente universitário. Pesq Prat Psicossociais. (2019):14(1);e1801. Available from: http://pepsic.bvsalud.org/pdf/ppp/ v14n1/16.pdf

15. Wolffenbüttel K, Carneiro N. Uma breve história dos Centros de Testagem e Aconselhamento (CTA) enquanto organização tecnológica de prevenção de DST/Aids no Brasil e no estado de São Paulo. Saúde Coletiva. 2007;4(18):183-187. Disponível em: https://www.redalyc.org/ pdf/842/84218406.pdf

16. Barros CRS, Zucchi EM, Schraiber LB, França Junior I. Individualand contextual-level factors associated with client-initiated HIV testing. Rev bras Epidemiol. 2017;20(3):394-407. doi: https://doi.org/10.1590/19805497201700030004

17. Campos CGAP, Estima SL, Santos VS, Lazzarotto AR. A 
vulnerabilidade ao HIV em adolescentes: estudo retrospectivo em um centro de testagem e aconselhamento. Rev Min Enferm. 2014;18(2):310-319. doi: https://doi.org/10.5935/1415-2762.20140024

18. Brito FG, Rezende MIRS, Madi RR, Melo CM. Perfil Epidemiológico de Portadores do Vírus da Imunodeficiêcia Humana e Síndrome da Imunodeficiência Adquirida no estado de Sergipe, 2007-2012. Interfaces Cient Sau Amb. 2014;2(2):59-71. doi: http://dx.doi.org/10.17564/2316$3798.2014 v 2 n 2 p 59-71$

19. Nogueira FJS, Callou Filho CR, Mesquita CAM, Souza ES, Saraiva AKM. Caracterização dos usuários atendidos em um centro de testagem e aconselhamento em infecções relacionadas ao sexo. Saude e pesqui. 2017; 10(2): 243-250, May-Aug. 2017. Disponível em: https://periodicos. unicesumar.edu.br/index.php/saudpesq/article/view/5861

20. Ferreira CO, Davoglio RS, Vianna ASA, Silva AA, Rezende REA, Davoglio TR. Vulnerabilidade a infecções sexualmente transmissíveis em idosos usuários de um centro de testagem e aconselhamento. Arq Cienc Saúde UNIPAR. 2019;23(3):171-180. http://dx.doi.org/10.25110/arqsaude. v23i3.2019.6757

21. Segundo FLF, Davoglio RS, Souza LM, Canário DDRC, Nascimento AAJ, Coelho GMP. Fatores associados às infecções sexualmente transmissíveis em usuários de centro de testagem e aconselhamento da Bahia. Rev. baiana saúde pública. 2017; 40(3). doi: https://doi.org/10.22278/23182660.2016.v40.n3.a1593.

22. Bassichetto KC, Mesquita F, Zacaro C, Santos EA, Oliveira SM. Perfil epidemiológico dos usuários de um Centro de Testagem e Aconselhamento para DST/HIV da Rede Municipal de São Paulo, com sorologia positiva para o HIV. Rev Bras Epidemiol. 2004;7(3):302-310. doi: https://doi.org/10.1590/ S1415-790X2004000300008

23. Soares PS, Brandão ER. Não retorno de usuários a um Centro de Testagem e Aconselhamento do Estado do Rio de Janeiro: fatores estruturais e subjetivos. Physis. 2013;23(3):703-721. doi: https://doi.org/10.1590/ S0103-73312013000300003

24. Matos MMM, Fernandes AKJ, Mallmann CSY, Menezes MP, Matos EL. Perfis sociocomportamentais dos usuários do Centro de Testagem e Aconselhamento-CTA em DST/aids do Hospital Universitário Getúlio Vargas da cidade de Manaus-AM. Rev HUGV. 2011;4(6):25-33. Available from: http:/hugv.ufam.edu.br/downloads/revistas/REVISTA\%202011/ Revista\%201\%20Artigos/artigo\%2002.pdf

25. Souza NR, Bernardes EH, Carmo TMD, Nascimento E, Silva ES, Souza BNA et al. Perfil da população idosa que procura o centro de referência em DST/Aids de Passos/MG. DST J Bras Doenças Sex Transm. 2011;23(4):198-204. doi: https://doi.org/10.5533/2177-8264-201123409

26. Araujo GM, Leite MT, Hildebrandt LM, Oliveski CC, Beuter M. Self-care of elderly people after the diagnosis of acquired immunodeficiency syndrome. Rev Bras Enferm. 2018;71(Suppl 2):793-800. doi: https://doi. org/10.1590/0034-7167-2017-0248.

27. Vilela MP, Brito TRP, Goyatá SLT, Arantes CIS. Perfil epidemiológico dos usuários do Centro de Testagem e Aconselhamento de Alfenas, Minas Gerais. Rev Eletr Enferm. 2010;12(2):326-30. doi: http://dx.doi.org/10.5216/ ree.v12i 2.5200

28. Barbosa AJC, Bertin F, Silva RMM, Faller JW, Silva-Sobrinho RA, Zilly A. Behavioral profile of pregnant women attended in a testing and counseling center in a frontier municipality. Cogitare enferm. 2016; 21(1): 01-08. Disponível em: http://docs.bvsalud.org/biblioref/2016/07/726/42957174051-1-pb.pdf 